\title{
Stem cell-based therapy for neonatal lung disease: it is in the juice
}

\author{
Moses E. Fung ${ }^{1}$ and Bernard Thébaud ${ }^{2,3}$
}

Bronchopulmonary dysplasia, the chronic lung disease of prematurity, is the most common complication in extremely premature infants (born before 28wk gestation). Despite advances in perinatal care, modern clinical management remains devoid of therapies specifically promoting lung repair and lung growth. Recent progress in stem cell biology has uncovered the promise of stem/progenitor cells to repair damaged organs. Contrary to the original theory that stem cells engraft and repopulate the damaged organ, evidence suggests that stem cells act via a paracrine mechanism. This review highlights the preclinical evidence for the therapeutic potential of cell-based therapies in animal models of neonatal chronic lung injury and the multiple therapeutic avenues offered by soluble stem cell-derived factors.

$\mathbf{T}$ he incidence of premature delivery in North America is $12.5 \%$ and rising (1). Prematurity is the leading cause of perinatal mortality and morbidity, placing these neonates at high risk for long-term medical impairments such as bronchopulmonary dysplasia (BPD). First documented in 1967 by Northway et al. (2), BPD was described as a chronic lung disease following mechanical ventilation and oxygen therapy for acute respiratory failure at birth. Since then, antenatal steroids and postnatal surfactant have aided in overcoming the biochemical immaturity of the lung. These advances in perinatal care, together with more incremental improvements, enabled neonatologists to push back the limits of viability from the previous 34 wk gestation to the current $\sim 24$ wk gestation. Injury to more immature lungs changed the pathology of $\operatorname{BPD}(3,4)$. Today, BPD is characterized by impaired alveolar development and dysmorphic pulmonary microvascular growth, along with a lesser degree of inflammation and fibrosis compared with the original BPD (5). Injury at these earlier stages may be more challenging to prevent and increases the risk of long-term consequences, including pulmonary hypertension $(\mathrm{PH})$ and earlyonset emphysema (6), which add to the burden of health care $(7,8)$. Thus, therapies that promote both lung repair and lung growth are desirable.

Recent insights into stem cell biology promise the regeneration of damaged organs. Stem cells are capable of self-renewal and differentiation into specialized cell types and thus have the potential to promote organogenesis, tissue regeneration, maintenance, and repair (9). Mesenchymal stromal cells (MSCs) attracted particular interest because of their ease of isolation, characterization, apparent multipotency, and pleiotropic effects. Adult bone marrow-derived MSCs (BMSCs) apparently differentiate into cells of various nonhematopoietic tissues. BMSC studies in various disease models, including cardiovascular and neurodegenerative disorders, demonstrated their efficacy in attenuating organ injury (10-13). The demonstration that a bone marrow-derived stem cell could differentiate into alveolar epithelial cells ignited stem cell research in the lung (14). Accordingly, preclinical studies suggested that bone marrow-derived stem/progenitor cells were capable of migrating to the injured lung to promote repair (15), and administration of exogenous BMSCs prevented lung injury in various adult lung disease models (reviewed by Weiss et al. (9)). These studies offered substantial promise to mitigate the impaired alveolar growth in experimental models mimicking BPD. The multipotency and self-renewal of stem cells make cell-based therapies appealing for providing both lung injury prevention and lung growth.

\section{CELL-BASED THERAPIES TO PREVENT EXPERIMENTAL} CHRONIC NEONATAL LUNG INJURY: PROOF OF CONCEPT

In 2007, Tian et al. (16) showed that intravenous injection of rat BMSCs could ameliorate neonatal lung injury. Shortly after, two simultaneously published articles confirmed the therapeutic potential of BMSCs. Intravenously delivered BMSCs reduced alveolar loss and lung inflammation and prevented PH in hyperoxia-induced mice (17). Likewise, intratracheal delivery of BMSCs increased survival and exercise capacity of hyperoxia-exposed rats while attenuating alveolar and vascular injury and PH (18). Subsequent studies also showed benefits in weight gain (19) and decreased fibrosis (20).

A clinically relevant source of stem cells, especially for the treatment of neonatal diseases, is offered by umbilical cord blood (UCB) (21). UCB contains stem cells that are easily accessible at birth and also capable of differentiating into various

'Women and Children's Health Research Institute, Cardiovascular Research Center and Pulmonary Research Group, University of Alberta, Edmonton, Alberta, Canada; ${ }^{2}$ Ottawa Hospital Research Institute, Regenerative Medicine Program, Sprott Centre for Stem Cell Research, Ottawa, Ontario, Canada; ${ }^{3}$ Department of Pediatrics, Children's Hospital of Eastern Ontario Research Institute, University of Ottawa, Ottawa, Ontario, Canada. Correspondence: Bernard Thébaud (bthebaud@ohri.ca) 


\section{Paracrine effect of stem cells for BPD $\quad$ ReView}

cell types (22-24), including alveolar epithelial cells (25). Intratracheal and intraperitoneal administration of human UCB-derived MSCs also improved alveolar growth through various mechanisms (26) and in a dose-dependent manner: $5 \times 10^{3}$ cells failed to attenuate both hyperoxia-induced lung injury and inflammation, whereas $5 \times 10^{4}$ and $5 \times 10^{5}$ cells attenuated both hyperoxia-induced injuries and inflammatory responses, but the latter dose was more effective (27). Human cord-derived pericytes and UCB-derived MSCs not only could prevent but also could repair lung injury in neonatal rats when administered $2 \mathrm{wk}$ after established hyperoxia-induced lung injury (28). Long-term assessment at 6 mo showed persistent improvement in lung architecture and exercise capacity, and no adverse effects were observed (28).

While MSCs are affirming their promise in regenerative medicine, other stem and progenitor cells are emerging. Amnion epithelial cells prevent antenatal lipopolysaccharideinduced (29) and ventilation-induced (30) lung injury in fetal sheep. Multipotent amniotic fluid-derived stem cells are capable of differentiating into lineages of all three embryonic germ layers and promote alveolar epithelial cell wound healing and lung growth $(31,32)$. Consistent with the importance of angiogenesis during lung growth, injury, and repair (33), bone marrow-derived angiogenic cells-a novel population of bone marrow myeloid progenitor cells that express angiogenic markers-demonstrated the capacity to restore impaired alveolar and vascular lung growth in hyperoxia-exposed newborn mice (34).

Overall, these observations (summarized in Table 1) provide evidence for the therapeutic benefit of bone marrow- and cord blood-derived MSCs in chronic oxygen-induced lung injury in rodents. A recurrent finding, however, is the paucity of cell engraftment, suggesting that stem cell properties such as self-renewal and differentiation are not required for their therapeutic action (35). This finding has led to the hypothesis that MSCs act through a paracrine effect (36), rather than through cell replacement. This realization has expanded the therapeutic options of cell-based therapies.

\section{MSCs PREVENT LUNG INJURY VIA A PARACRINE MECHANISM: IT IS IN THE JUICE}

Several lines of evidence suggest that MSCs act via a paracrine mechanism to protect the developing lung from injury. In vitro, cell-free BMSC-derived conditioned media prevented hyperoxia-induced alveolar epithelial cell apoptosis, accelerated alveolar epithelial cell wound healing, and preserved endothelial cord formation on matrigel during hyperoxia (18).

In vivo, the paracrine effect could also be inferred from the efficacy of intraperitoneal administration of MSCs in preventing oxygen-induced neonatal lung injury $(20,26)$. Accordingly, Aslam et al. (17) provided direct in vivo evidence showing that a single injection of cell-free BMSC-derived conditioned media had a more pronounced effect on alveolar injury and fibrosis prevention than that of BMSCs themselves. In a follow-up study, a single intravenous dose of BMSC-derived conditioned media normalized lung function and reversed alveolar injury and $\mathrm{PH}$ (37). A single intratracheal injection of BMSC or BMSC-free conditioned media protected from oxygen-induced alveolar and vascular injury with a persistent benefit followed up to 3 mo (38). Likewise, cell-free conditioned media derived from human UCB-MSCs and pericytes prevented and reversed arrested alveolar growth and lung function in hyperoxia-exposed rats with persistent benefits at 6 mo of age and without adverse effect on lung structure or tumor formation (28). Dose-response studies have not yet been performed.

Although the therapeutic benefit of the conditioned media is undeniable, a potential caveat of this strategy is the lack of cell adaptation to the local injurious environment. In an attempt to overcome this potential limitation, Waszak et al. (39) exposed BMSCs to a "deleterious BPD environment" by priming them ex vivo in hyperoxia for $24 \mathrm{~h}$. Conditioned media collected from these preconditioned cells and injected into hyperoxic rats exerted a more potent therapeutic effect in vivo on lung architecture as compared with nonpreconditioned media.

Thus, rather than replacing injured cells and differentiating into lung cells, MSCs may release factors that protect resident lung cells from injury or modulate the function of inflammatory cells. Tropea et al. recently provided evidence for such a scenario. Bronchioalveolar stem cells are putative epithelial lung stem/progenitor cells at the bronchioalveolar junction, capable of self-renewal and differentiation in culture and also proliferate in response to alveolar injury (40). Both BMSC and BMSC-derived conditioned media increase the number of bronchioalveolar stem cells in neonatal mice exposed to hyperoxia (41). This study also offers new therapeutic perspectives, i.e., the protection of resident lung progenitor cells rather than exogenous supplementation of stem cells. In addition, there is increasing evidence that MSCs interact with inflammatory cells to modulate their response to injury. MSCs can direct macrophages from a M1 (proinflammatory) to a M2 (healer) phenotype in various disease conditions $(42,43)$. Overall, these observations suggest that cell-free conditioned media exerts similar therapeutic benefit to the cell itself. The exciting challenge is how to harness the multiple healing properties of stem cells.

\section{MSCS PREVENT LUNG INJURY VIA A PARACRINE MECHANISM: WHAT IS IN THE JUICE?}

Indeed, the identification of soluble factors in the conditioned media may allow the discovery of novel healing molecules that each by itself or in combination could yield new therapeutic options (44). Besides factors already known to be lung protective, including keratinocyte growth factor (45), vascular endothelial growth factor (46), or adiponectin (47), novel molecules secreted by MSCs have already been identified and shown therapeutic benefit in various disease models, such as stanniocalcin-1 (48)-a potent antioxidant-or tumor necrosis factor- $\alpha$-stimulated gene/protein 6 (TSG-6) (49) -a potent anti-inflammatory protein.

From a clinical perspective, a relevant question for the design of clinical trials is to determine the most efficacious 
Table 1. Stem/progenitor cell preclinical trials in experimental neonatal lung diseases

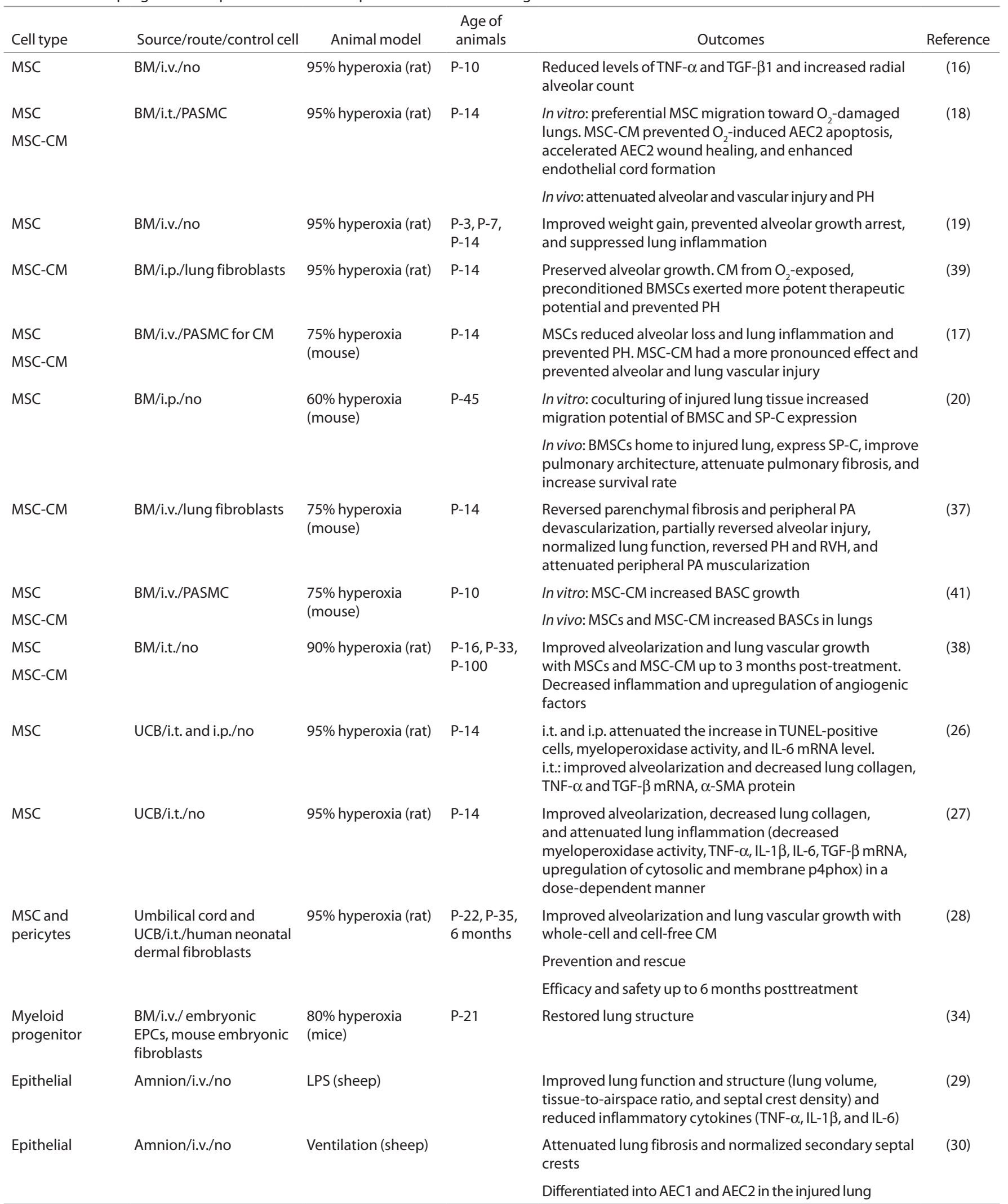

AEC2, alveolar epithelial type II cell; BASC, bronchioalveolar stem cell; BM, bone marrow; BMSC, bone marrow-derived MSC; CM, conditioned media; dUTP, deoxyuridine triphosphate; EPC, endothelial progenitor cell; IL, interleukin; i.p., intraperitoneal; i.t., intratracheal; i.v., intravenous; LPS, lipopolysaccharide; MSC, mesenchymal stromal cell; P, postnatal day; PA, pulmonary artery; PASMC, PA smooth muscle cell; PH, pulmonary hypertension; RVH, right ventricular hypertrophy; SMA, smooth muscle actin; SP-C, surfactant protein C; TGF, transforming growth factor; TNF, tumor necrosis factor; TUNEL, terminal deoxynucleotidyl transferase dUTP nick end labeling; UCB, umbilical cord blood. 


\section{Paracrine effect of stem cells for BPD $\quad$ Review}

and safest stem cell-based approach: whole-cell therapy vs. cell-free conditioned media vs. identification of single bioactive molecules vs. identification and determination of the most efficacious combination of molecules. This daunting task could be circumvented by the recent recognition that MSCs release membrane vesicles, exosomes in particular, that act as nanopackages containing a combination of bioactive molecules and microRNAs (50). microRNAs are small noncoding RNA molecules involved in transcriptional regulation of gene expression. In particular, microRNAs could become interesting therapeutic targets in the prevention of BPD (51) by silencing specific genes with deleterious effects during lung injury. Exosomes are $40-100 \mathrm{~nm}$ in size and represent a specific subtype of secreted membrane vesicles formed through the fusion of multivesicular endosomes with the plasma membrane. Although known for several decades, membrane vesicles have long been thought of as mere cell debris. Recent evidence, however, suggests that MSC-derived exosomes play important roles in cell communication and mediate the therapeutic benefit of MSCs. For example, MSC-derived exosomes attenuate lung macrophage influx, decrease proinflammatory cytokine levels in the bronchoalveolar lavage, and prevent pulmonary vascular remodeling and hypoxia-induced $\mathrm{PH}$ in mice (52). With the exosomes removed, the conditioned media showed no therapeutic effect in this model. Similar therapeutic benefits of MSC-derived exosomes are reported in kidney (53) and cardiac (54) injury. A limitation for the exploitation of exosomes as therapeutic tools remains the process of isolation, characterization, quality control, and large-scale manufacturing. Novel findings continue to uncover the mechanisms by which MSCs protect resident lung cells including the transfer of mitochondria via nanotubes (55). These pleiotropic effects (Figure 1) open exciting avenues, in particular, for multifactorial diseases such as BPD and provide traction for the discoveries of cell-free products.

\section{CONSIDERATIONS FOR CLINICAL TRIALS}

A clinical trial testing the safety and efficacy of MSCs in adult patients with chronic obstructive pulmonary disease has been completed (56). Although this clinical trial was predominantly for safety, no substantial evidence of efficacy of the MSCs was recorded. More recently, a phase I clinical trial testing the safety of human UCB-derived MSCs in nine premature infants at risk of developing BPD has been completed (ClinicalTrials. gov: NCT01297205). Although this study upholds the safety of MSC therapy, long-term follow-up is warranted. Further clinical trials are already planned (ClinicalTrials.gov: NCT01207869, NCT01828957).

Preclinical studies have generated proof of concept evidence that cell-based therapies can prevent and restore experimental neonatal lung injury in rodents and sheep. Rather than cell replacement, the therapeutic benefit of stem cells is mediated through a paracrine effect. It is likely that the combination of bioactive molecules contained in the conditioned media provides the compounding pleiotropic effects attributed to MSCs. Administration of the entire cocktail containing unidentified products may conjure unforeseen side effects, and some components may be more beneficial in repair. Thus, further specification of which molecules have reparative properties and/or the isolation of specific micro/nano carriers such as exosomes may lead to pharmacological therapies for BPD.

The cell most suitable for clinical trials appears to be MSCs, likely because of their ease of isolation, characterization, and pleiotropic effects. However, endothelial progenitor cells and other stem/progenitor cells have also proven to be effective in preclinical BPD models. These various cells differ in their roles and respective factors, thereby possibly producing a more pronounced effect when administered in concert (57), although this remains to be proven in the lung.

Likewise, the source of cells is an important consideration. Umbilical cord and cord blood are easily accessible at birth and may have more potent repair capabilities than that of adult

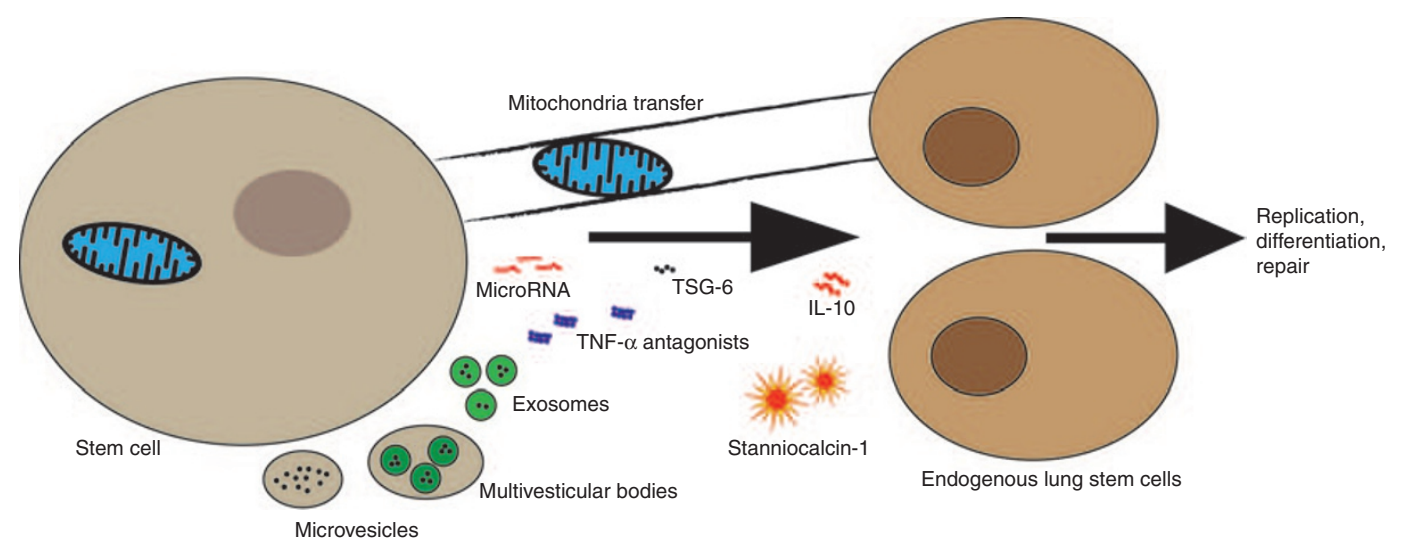

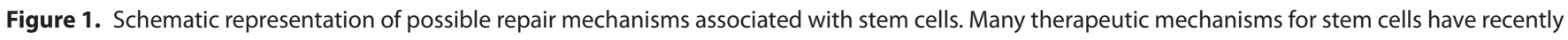
emerged. These include microparticle carriers such as microvesicles, exosomes, or multivesicular bodies, which are speculated to be released by stem cells and elicit a therapeutic response. MicroRNA packaged in these vesicles or as a sole effector may also play a therapeutic role. The role of secreted soluble proteins/peptides in neonatal and adult lung injury has been extensively studied. This has lead to the discovery of promising bioactive molecules such as the anti-inflammatory interleukin-10 (IL-10), stanniocalcin-1, tumor necrosis factor- $\alpha$-stimulated gene/protein 6 (TSG-6), and tumor necrosis factor- $\alpha$ (TNF- $\alpha$ ) antagonists, the combination of which may contribute to the pleiotropic effects promoting repair. Recent evidence also unveiled therapeutic mitochondria transfer via nanotubes. These mechanisms can signal endogenous stem cells to amplify or transduce similar repair actions. 
BMSCs. Autologous UCB-derived cell therapies may avoid immunological risks and allows the use of minimally manipulated cells. However, given the immunological properties of MSCs, allogeneic cell therapy is feasible and may facilitate the logistics of cell-based therapies.

The timing of the treatment is another factor to be resolved. Recent preclinical evidence showed that UCB-derived MSCs time dependently attenuated hyperoxia-induced injury, eliciting significant protection in the early, but not the late, phase of inflammation (58). In the clinic, the early identification of infants at the most risk of developing BPD through the use of estimators and models may allow for the selection of an appropriate patient population. Patients with early and persistent pulmonary dysfunction have a $\sim 70 \%$ risk of developing BPD, as defined by Laughon et al. (59) and may represent an at-risk population of choice for cell-based therapies.

Finally, the safety of each of these cell-based therapies must be investigated thoroughly in well-designed preclinical trials, including large animal models.

In summary, as the incidence of prematurity and chronic neonatal lung disease rises (60), novel therapies are required. Preclinical studies have brought substantial promise in developing an effective clinical therapy that could fulfill the dual role of preventing injury and promoting lung growth. The paracrine effect of cell-based therapies has opened unexpected therapeutic options through the identification of individual molecules or mechanisms including microRNAs, mitochondrial transfer, and microparticles. The promise may not lie in the stem cell itself, but rather in its vast array of bioactive mediators-"it is in the juice."

\section{STATEMENT OF FINANCIAL SUPPORT}

B.T. is supported by the Canadian Institute of Health Research, the Ottawa Hospital Research Institute, and the Children's Hospital of Eastern Ontario Research Institute. M.E.F. is supported by a summer studentship from Alberta Innovates Health Solutions.

Disclosure: The authors have nothing to disclose.

\section{REFERENCES}

1. Goldenberg RL, Culhane JF, Iams JD, Romero R. Epidemiology and causes of preterm birth. Lancet 2008;371:75-84.

2. Northway WH Jr, Rosan RC, Porter DY. Pulmonary disease following respirator therapy of hyaline-membrane disease. Bronchopulmonary dysplasia. N Engl J Med 1967;276:357-68.

3. Jobe AH, Bancalari E. Bronchopulmonary dysplasia. Am J Respir Crit Care Med 2001;163:1723-9.

4. Jobe AJ. The new BPD: an arrest of lung development. Pediatr Res 1999;46:641-3.

5. Baraldi E, Filippone M. Chronic lung disease after premature birth. N Engl J Med 2007;357:1946-55.

6. Saigal S, Doyle LW. An overview of mortality and sequelae of preterm birth from infancy to adulthood. Lancet 2008;371:261-9.

7. Ireys HT, Anderson GF, Shaffer TJ, Neff JM. Expenditures for care of children with chronic illnesses enrolled in the Washington State Medicaid program, fiscal year 1993. Pediatrics 1997;100(2 Pt 1):197-204.

8. Wong PM, Lees AN, Louw J, et al. Emphysema in young adult survivors of moderate-to-severe bronchopulmonary dysplasia. Eur Respir J 2008;32:321-8.

9. Weiss DJ, Bertoncello I, Borok Z, et al. Stem cells and cell therapies in lung biology and lung diseases. Proc Am Thorac Soc 2011;8:223-72.

10. Pereira RF, O'Hara MD, Laptev AV, et al. Marrow stromal cells as a source of progenitor cells for nonhematopoietic tissues in transgenic mice with a phenotype of osteogenesis imperfecta. Proc Natl Acad Sci USA 1998;95:1142-7.
11. Shah RV, Mitchell RN. The role of stem cells in the response to myocardial and vascular wall injury. Cardiovasc Pathol 2005;14:225-31.

12. Phinney DG, Isakova I. Plasticity and therapeutic potential of mesenchymal stem cells in the nervous system. Curr Pharm Des 2005;11:1255-65.

13. Phinney DG, Prockop DJ. Concise review: mesenchymal stem/multipotent stromal cells: the state of transdifferentiation and modes of tissue repaircurrent views. Stem Cells 2007;25:2896-902.

14. Krause DS, Theise ND, Collector MI, et al. Multi-organ, multi-lineage engraftment by a single bone marrow-derived stem cell. Cell 2001;105: $369-77$.

15. Griffiths MJ, Bonnet D, Janes SM. Stem cells of the alveolar epithelium. Lancet 2005;366:249-60.

16. Tian ZF, DU J, Wang B, Hong XY, Feng ZC. [Intravenous infusion of rat bone marrow-derived mesenchymal stem cells ameliorates hyperoxiainduced lung injury in neonatal rats]. Nan Fang Yi Ke Da Xue Xue Bao 2007;27:1692-5.

17. Aslam M, Baveja R, Liang OD, et al. Bone marrow stromal cells attenuate lung injury in a murine model of neonatal chronic lung disease. Am J Respir Crit Care Med 2009;180:1122-30.

18. van Haaften T, Byrne R, Bonnet S, et al. Airway delivery of mesenchymal stem cells prevents arrested alveolar growth in neonatal lung injury in rats. Am J Respir Crit Care Med 2009;180:1131-42.

19. Zhang H, Fang J, Su H, et al. Bone marrow mesenchymal stem cells attenuate lung inflammation of hyperoxic newborn rats. Pediatr Transplant 2012;16:589-98.

20. Zhang X, Wang H, Shi Y, et al. Role of bone marrow-derived mesenchymal stem cells in the prevention of hyperoxia-induced lung injury in newborn mice. Cell Biol Int 2012;36:589-94.

21. McGuckin CP, Forraz N. Umbilical cord blood stem cells-an ethical source for regenerative medicine. Med Law 2008;27:147-65.

22. Hou L, Cao H, Wang D, et al. Induction of umbilical cord blood mesenchymal stem cells into neuron-like cells in vitro. Int J Hematol 2003;78:256-61.

23. Lee OK, Kuo TK, Chen WM, Lee KD, Hsieh SL, Chen TH. Isolation of multipotent mesenchymal stem cells from umbilical cord blood. Blood 2004;103:1669-75.

24. Kögler G, Sensken S, Airey JA, et al. A new human somatic stem cell from placental cord blood with intrinsic pluripotent differentiation potential. J Exp Med 2004;200:123-35.

25. Berger MJ, Adams SD, Tigges BM, et al. Differentiation of umbilical cord blood-derived multilineage progenitor cells into respiratory epithelial cells. Cytotherapy 2006;8:480-7.

26. Chang YS, Oh W, Choi SJ, et al. Human umbilical cord blood-derived mesenchymal stem cells attenuate hyperoxia-induced lung injury in neonatal rats. Cell Transplant 2009;18:869-86.

27. Chang YS, Choi SJ, Sung DK, et al. Intratracheal transplantation of human umbilical cord blood-derived mesenchymal stem cells dose-dependently attenuates hyperoxia-induced lung injury in neonatal rats. Cell Transplant 2011;20:1843-54.

28. Pierro M, Ionescu L, Montemurro T, et al. Short-term, long-term and paracrine effect of human umbilical cord-derived stem cells in lung injury prevention and repair in experimental bronchopulmonary dysplasia. Thorax 2013;68:475-84.

29. Vosdoganes P, Hodges RJ, Lim R, et al. Human amnion epithelial cells as a treatment for inflammation-induced fetal lung injury in sheep. Am J Obstet Gynecol 2011;205:156.e26-33.

30. Hodges R, Jenkin G, Hooper SB, et al. Human amnion epithelial cells reduce ventilation-induced preterm lung injury in fetal sheep. Am J Obstet Gynecol 2012;206:448.e8-15.

31. Buckley S, Shi W, Carraro G, et al. The milieu of damaged alveolar epithelial type 2 cells stimulates alveolar wound repair by endogenous and exogenous progenitors. Am J Respir Cell Mol Biol 2011;45:121221.

32. Pederiva F, Ghionzoli M, Pierro A, De Coppi P, Tovar JA. Amniotic fluid stem cells rescue both in vitro and in vivo growth, innervation, and motility in nitrofen-exposed hypoplastic rat lungs through paracrine effects. Cell Transplant 2013;22:1683-94. 


\section{Paracrine effect of stem cells for BPD $\quad$ ReView}

33. Thébaud B, Abman SH. Bronchopulmonary dysplasia: where have all the vessels gone? Roles of angiogenic growth factors in chronic lung disease. Am J Respir Crit Care Med 2007;175:978-85.

34. Balasubramaniam V, Ryan SL, Seedorf GJ, et al. Bone marrow-derived angiogenic cells restore lung alveolar and vascular structure after neonatal hyperoxia in infant mice. Am J Physiol Lung Cell Mol Physiol 2010;298:L315-23.

35. Bianco P, Cao X, Frenette PS, et al. The meaning, the sense and the significance: translating the science of mesenchymal stem cells into medicine. Nat Med 2013;19:35-42.

36. Lee JW, Fang X, Krasnodembskaya A, Howard JP, Matthay MA. Concise review: mesenchymal stem cells for acute lung injury: role of paracrine soluble factors. Stem Cells 2011;29:913-9.

37. Hansmann G, Fernandez-Gonzalez A, Aslam M, et al. Mesenchymal stem cell-mediated reversal of bronchopulmonary dysplasia and associated pulmonary hypertension. Pulm Circ 2012;2:170-81.

38. Sutsko RP, Young KC, Ribeiro A, et al. Long-term reparative effects of mesenchymal stem cell therapy following neonatal hyperoxia-induced lung injury. Pediatr Res 2013;73:46-53.

39. Waszak P, Alphonse R, Vadivel A, Ionescu L, Eaton F, Thébaud B. Preconditioning enhances the paracrine effect of mesenchymal stem cells in preventing oxygen-induced neonatal lung injury in rats. Stem Cells Dev 2012;21:2789-97.

40. Kim CF, Jackson EL, Woolfenden AE, et al. Identification of bronchioalveolar stem cells in normal lung and lung cancer. Cell 2005;121:823-35.

41. Tropea KA, Leder E, Aslam M, et al. Bronchioalveolar stem cells increase after mesenchymal stromal cell treatment in a mouse model of bronchopulmonary dysplasia. Am J Physiol Lung Cell Mol Physiol 2012;302:L829-37.

42. Ionescu L, Byrne RN, van Haaften T, et al. Stem cell conditioned medium improves acute lung injury in mice: in vivo evidence for stem cell paracrine action. Am J Physiol Lung Cell Mol Physiol 2012;303:L967-77.

43. Németh $\mathrm{K}$, Leelahavanichkul A, Yuen PS, et al. Bone marrow stromal cells attenuate sepsis via prostaglandin $\mathrm{E}(2)$-dependent reprogramming of host macrophages to increase their interleukin-10 production. Nat Med 2009;15:42-9.

44. Caplan AI, Correa D. The MSC: an injury drugstore. Cell Stem Cell 2011;9:11-5.

45. Lee JW, Fang X, Gupta N, Serikov V, Matthay MA. Allogeneic human mesenchymal stem cells for treatment of E. coli endotoxin-induced acute lung injury in the ex vivo perfused human lung. Proc Natl Acad Sci USA 2009;106:16357-62.

46. Thébaud B, Ladha F, Michelakis ED, et al. Vascular endothelial growth factor gene therapy increases survival, promotes lung angiogenesis, and prevents alveolar damage in hyperoxia-induced lung injury: evidence that angiogenesis participates in alveolarization. Circulation 2005;112:2477-86.

47. Ionescu LI, Alphonse RS, Arizmendi N, et al. Airway delivery of soluble factors from plastic-adherent bone marrow cells prevents murine asthma. Am J Respir Cell Mol Biol 2012;46:207-16.

48. Block GJ, Ohkouchi S, Fung F, et al. Multipotent stromal cells are activated to reduce apoptosis in part by upregulation and secretion of stanniocalcin-1. Stem Cells 2009;27:670-81.

49. Lee RH, Pulin AA, Seo MJ, et al. Intravenous hMSCs improve myocardial infarction in mice because cells embolized in lung are activated to secrete the anti-inflammatory protein TSG-6. Cell Stem Cell 2009;5:54-63.

50. Chaput N, Théry C. Exosomes: immune properties and potential clinical implementations. Semin Immunopathol 2011;33:419-40.

51. Dong J, Carey WA, Abel S, et al. MicroRNA-mRNA interactions in a murine model of hyperoxia-induced bronchopulmonary dysplasia. BMC Genomics 2012;13:204.

52. Lee C, Mitsialis SA, Aslam M, et al. Exosomes mediate the cytoprotective action of mesenchymal stromal cells on hypoxia-induced pulmonary hypertension. Circulation 2012;126:2601-11.

53. Biancone L, Bruno S, Deregibus MC, Tetta C, Camussi G. Therapeutic potential of mesenchymal stem cell-derived microvesicles. Nephrol Dial Transplant 2012;27:3037-42.

54. Lai RC, Chen TS, Lim SK. Mesenchymal stem cell exosome: a novel stem cell-based therapy for cardiovascular disease. Regen Med 2011;6:481-92.

55. Islam MN, Das SR, Emin MT, et al. Mitochondrial transfer from bonemarrow-derived stromal cells to pulmonary alveoli protects against acute lung injury. Nat Med 2012;18:759-65.

56. Weiss DJ, Casaburi R, Flannery R, Leroux-Williams M, Tashkin DP. A placebo-controlled randomized trial of mesenchymal stem cells in chronic obstructive pulmonary disease. Chest 2013;143:1590-8.

57. Aguirre A, Planell JA, Engel E. Dynamics of bone marrow-derived endothelial progenitor cell/mesenchymal stem cell interaction in co-culture and its implications in angiogenesis. Biochem Biophys Res Commun 2010;400:284-91.

58. Chang YS, Choi SJ, Ahn SY, et al. Timing of umbilical cord blood derived mesenchymal stem cells transplantation determines therapeutic efficacy in the neonatal hyperoxic lung injury. PLoS ONE 2013;8:e52419.

59. Laughon M, Allred EN, Bose C, et al.; ELGAN Study Investigators. Patterns of respiratory disease during the first 2 postnatal weeks in extremely premature infants. Pediatrics 2009;123:1124-31.

60. Shah PS, Sankaran K, Aziz K, et al.; Canadian Neonatal Network. Outcomes of preterm infants $<29$ weeks gestation over 10-year period in Canada: a cause for concern? J Perinatol 2012;32:132-8. 